\title{
Retos docentes en la didáctica de traducción: diseño y evaluación de la enseñanza de la localización de aplicaciones móviles
}

\author{
Challenges in Translator Training: Design and Evaluation of a Mobile App Localization Module \\ Desafios de professores em didática de tradução: design e avaliação do ensino de localização \\ de aplicativos móveis
}

María del Mar Sánchez Ramos* (https://orcid.org/0000-0001-5684-1552)

Departamento de Filología Moderna, Universidad de Alcalá, España
Recibido: 29-09-17

Revisado: $20-11-17$

Aceptado: $30-11-17$

Publicado: $18-12-17$
RESUMEN. Conscientes del desarrollo imparable de la localización en el mercado traductológico, este artículo tiene como objetivo ofrecer una propuesta docente de enseñanza de aplicaciones móviles dentro del aula de traducción. Con tal fin se describe la actividad docente llevada a cabo en la asignatura de Localización (Grado en Lenguas Modernas y Traducción, Universidad de Alcalá, Españal con un grupo de 24 alumnos. Bajo una metodología de corte socioconstructivista se recogió información en una sesión grupal mediante un cuestionario, lo que proporcionó datos cualitativos para evaluar la propuesta docente. Los resultados obtenidos muestran que los principales desafíos de este tipo de localización en el aula son de índole lingüístico y técnico, y que su enseñanza se hace cada vez más necesaria si queremos preparar a nuestros estudiantes ante los nuevos retos de la industria de la traducción.
Palabras clave:

teléfono móvil, didáctica de la traducción, tecnología

ABSTRACT. Aware of the unstoppable advance of localization and its influence on

Keywords: mobile phone, 
module conducted with 24 undergraduate translator trainees as part of the Localization course (Degree in Modern Languages and Translation, University of Alcalá, Spain). Following a socio-constructivist methodology, qualitative data were collected via a group session and

translator

training,

technology a questionnaire, which allowed evaluating the teaching proposal. Upon examination of the results, we can state that the main challenges are related to linguistic and technical issues and that their training is a must if we want our students to be ready for what comes ahead.

RESUMO. Consciente do incessante desenvolvimento da localização no mercado de

Palavraschave: telefone celular, didáctica de tradução, tecnologia metodologia socioconstrutivista, a informação foi coletada em uma sessão de grupo através de um questionário, que forneceu dados qualitativos para avaliar a proposta de ensino. Os resultados mostram que os principais desafios deste tipo de localização na sala de aula são de natureza linguística e técnica e que seu ensino torna-se cada vez mais necessário se quisermos preparar nossos alunos antes dos novos desafios do setor de tradução.

\section{RETOS DOCENTES EN LA DIDÁCTICA DE TRADUCCIÓN: DISEÑO Y EVALUACIÓN DE LA ENSEÑANZA DE LA LOCALIZACIÓN DE APLICACIONES MÓVILES ${ }^{1}$}

Vivimos un momento en el que la desconexión digital es prácticamente imposible de obviar. Muchas de nuestras actividades diarias hacen uso de la tecnología y todo (o casi todol se puede conseguir con un clic, desde tareas puramente personales (i.e. envío de un correo) a otras de índole administrativa. Dentro de las distintas tecnologías, la comercialización de productos digitales como los teléfonos móviles, también llamados inteligentes, se encuentra en su punto más álgido y, por ende, las aplicaciones diseñadas para ellos se están convirtiendo en los productos estrella del mercado digital. Ray (2016) aporta un dato abrumador para el 2017: "With a billion tablets already online and four billion smartphones predicted to be in use worldwide by 2017, mobile is the next frontier for companies to implement and fine-tune the global customer experience" (p. 31). Y es que el teléfono móvil representa más que un medio de comunicación, ya que nos ofrece multitud de servicios y canales de ocio a través de la infinidad de aplicaciones disponibles. Además, somos testigos en estos últimos años de una venta al alza de estos productos, que se han extendido en el mercado internacional (Fundación Telefónica, 2015). Parte del éxito de su comercialización se debe precisamente a la venta de los mismos en un gran

\footnotetext{
1 La investigación descrita en este artículo se enmarca dentro del grupo de innovación docente “Herramientas Informáticas para la Traducción e Interpretación y su docencia-HITID, (UAH-GI14-71), Universidad de Alcalá (Madrid, España).
} 
número de países, destacando aquellos del continénte asiático, como China o Corea del Sur (Schäler, 2012), pues de esta forma aumenta el número de usuarios potenciales.

Este momento de globalización también afecta al ámbito educativo y los docentes en traducción hemos de ser conscientes de los cambios y demandas profesionales que se producen para mejor adaptarlos a las aulas. En aras de conocer el mercado profesional en cuanto a traducción y avances tecnológicos, el grupo OPTIMALE realizó un estudio entre un total de 738 proveedores de servicios lingüísticos pertenecientes a un amplio abanico geográfico (Valero-Garcés \& Toudic, 2015, p. 187). Uno de los objetivos era el de analizar las demandas tecnológicas de los participantes en dicha encuesta. De las distintas destrezas que las empresas manifestaban tener en cuenta como requisito a la hora de aumentar su plantilla se señalaba la importancia y la demanda que estaba adquiriendo la tecnología móvil, junto con la habilidad de localizar sitios web o programas informáticos. Los autores, en este sentido, advierten de que la formación de traductores no puede ignorar el mensaje que se trasmite en los resultados obtenidos en dicha encuesta (Valero-Garcés \& Toudic, 2015, p. 193).

La industria de la localización de aplicaciones móviles se ha convertido en los últimos años en un mercado en expansión como medio de comunicación digital, en el que el término localización se entiende como la adaptación lingüística y cultural de material digital a las necesidades de un mercado concreto (Schäler, 2011). Este campo profesional abre un abanico de posibilidades para los traductores como agentes imprescindibles en el proceso de localización. Nuestros egresados han de ser conscientes de las necesidades profesionales que pueden encontrar, así como de las destrezas y conocimientos que va a necesitar para realizar con éxito las tareas asociadas a la localización. La enseñanza de la localización, y en concreto la localización de aplicaciones móviles, conlleva el desarrollo de competencias que van más allá del plano lingüístico debido al propio proceso de localización y las características del producto de la localización. En nuestra opinión, la formación en este ámbito se presenta ineludible y se hace necesario integrar una formación específica en esta materia que contribuya a la profesionalización de nuestros egresados.

El principal objetivo de este trabajo es exponer una propuesta docente de enseñanza de aplicaciones móviles dentro del aula de traducción. Con tal fin se describe la actividad docente llevada a cabo en la asignatura de Localización (Grado en Lenguas Modernas y Traducción, Universidad de Alcalá, España) con un total de 24 alumnos. La primera parte del artículo contextualiza el estudio y ofrece una panorámica teórica de la localización, y más concretamente de la localización de aplicaciones móviles, y sus principales características. Seguidamente se expone el diseño del estudio y su metodología socioconstructivista. Se concluye con los resultados cualitativos obtenidos.

\section{LA LOCALIZACIÓN DE APLICACIONES MÓVILES: RETOS LINGÜÍSTICOS, TECNOLÓGICOS Y CULTURALES}

El desarrollo de la llamada World Wide Web (WWW) en los años 90 y los cambios políticos y económicos acaecidos en la sociedad han llevado a un desarrollo y aumento considerable del contenido que debe ser localizado, es decir, adaptado desde el punto de vista lingüístico y cultural a las necesidades de un determinado mercado. El nacimiento de la localización, o la industria de la localización, se remonta 
a finales de los años 70 y principios de los años 80 , cuando compañías tecnológicas, principalmente estadounidenses, comienzan a exportar a mercados extranjeros. Los siguientes años supusieron la consolidación de la industria de la localización. No obstante, a pesar de la importancia de esta disciplina y sus conexiones innegables con el mundo de la traducción, las investigaciones en este ámbito son escasas y la localización "remains a "little-known and poorly understood phenomenon outside of the relatively closed circle of its clients and practitioners" (Dunne, 2006, p. 1).

Existen términos claves al hablar de localización, y que creemos necesarios mencionar de forma breve para ofrecer una mejor contextualización de nuestro estudio. La palabra localización viene del nombre en inglés locale, que hace referencia a una combinación específica de lenguas, regiones y codificación de caracteres (Esselink, 2000, p. 1). Un determinado locale engloba “all regional standards supported by a software product, such as date/time formats, sorting standards, currencies, and character sets" (Somers, 2003, p. 67). Podemos tomar como ejemplo el español de México o el español de Argentina. El término localización también suele ir acompañado del acrónico GILT, que responde a los términos de Globalization, Internationalisation, Localization y Translation. Este acrónimo se utiliza en la industria de la localización para hacer referencia a una serie de procesos que, de forma conjunta, asegurará el éxito del producto.

Dejando a un lado la definición de traducción por cuestiones obvias, nos detendremos brevemente en el resto de términos. Muchos autores han proporcionado definiciones del término localización. A nuestro entender, la ofrecida por (Schäler, 2011) nos parece la más acertada por englobar los aspectos esenciales que intervienen en el proceso de localización, que queda definida como "the linguistic and cultural adaptation of digital content to the requirements and locale of a foreign market, and the provision of services and technologies for the management of multilingualism across the digital global information flow" (p. 157). Por otro lado, la internacionalización, un concepto más técnico “allows for translation into other languages without the need for re-design or re-compilation" (Esselink, 2000, p. 1). En otras palabras, la tarea de la internacionalización está relacionada con el diseño de un producto digital de tal forma que pueda ser localizado fácilmente. Por último, la globalización se acerca más a tareas empresariales y de márketing, tareas y actividades que harán que un producto pueda distribuirse en los mercados internacionales.

Sin duda, la comercialización de productos digitales tiene como consecuencia que la localización se haya convertido en una tarea imprescindible, que se abra paso en el ámbito académico, y, aunque de forma lenta, cada vez son más los programas de grado y posgrado que se centran en la enseñanza de la localización (Altanero, 2006; Baños \& Toto, 2013; Veiga Díaz, 2013). No obstante, y a pesar de estos esfuerzos, la localización no goza de la popularidad que las necesidades del mercado demandan más allá de aquellos que están en contacto directo con el mundo profesional de la localización como clientes y empresas (Drugan, 2013, p. 99). Hablar de localización nos acerca a distintos tipos de adaptación lingüística y cultural, según los distintos productos digitales (sitio web, producto informático, videojuego o aplicación móvil). Distinguir los tipos de localización según el producto localizado puede resultar confuso, ya que se comparten características en el proceso de localización y los distintos productos, con sus especificidades, suelen presentar características afines (por ejemplo, la localización de imágenes puede encontrarse tanto en la localización de un videojuego como en la de un sitio web). 
Es por ello, que retomamos la clasificación de Mata Pastor (2005), que distingue entre localización de productos informáticos (LPI) y localización de contenido web (LCW). La primera de ellas, y en la que a nuestro parecer se incluye la localización de aplicaciones móviles, engloba programas informáticos y “cualquier producto que quepa incluir genéricamente dentro del concepto de software, como las utilizadas, los juegos informáticos, los productos multimedia con fines educativos o de entretenimiento, etc., además de toda la documentación, electrónica e impresa, que los acompaña" (p. 190). Por otro lado, la LCW incluiría aquellos productos que han sido creados, albergados o procesados por alguna de las tecnologías relacionadas con la World Wide Web. Si bien, y como afirma este autor, la fusión de las tecnologías hace a veces difícil una separación y clasificación exhaustiva.

Un dispositivo móvil se define como un aparato de pequeño tamaño, con algunas capacidades de procesamiento, con conexión permanente o intermitente a una red, con memoria limitada, y multifuncional (Guevara Soriano, 2010). De acuerdo con esta definición existen multitud de dispositivos móviles, desde los reproductores de audio portátiles hasta los navegadores GPS, pasando por los teléfonos móviles y las tabletas. En este trabajo nos centraremos fundamentalmente en los teléfonos móviles, uno de los dispositivos móviles cuya adquisición y uso ha aumentado más en los últimos años (Fundación Telefónica, 2015). Los teléfonos móviles cuentan con las conocidas aplicaciones móviles, es decir, programas informáticos diseñados con un fin específico y que son ejecutados por los usuarios en dichos dispositivos, entre otros como tabletas (Lachat Leal, 2015).

La localización de aplicaciones móviles es un fenómeno reciente si lo comparamos con la localización de programas informáticos o localización de contenido web. La diversidad lingüística se observa también en el contexto de la localización, donde cada vez aumenta el número de lenguas consideradas minoritarias que escalan puestos en los mercados internacionales desbancando, de este modo, al inglés. Como afirma Schäler (2012), cada vez es mayor el número de aplicaciones móviles que se diseñan en lenguas distintas al inglés, lo que hace necesaria la localización de las mismas con el fin de generar beneficios para las compañías y abre, por consiguiente, el mercado profesional para los traductores. Según estos datos, el $50 \%$ de los países que ocupan los primeros diez puestos en descargas e ingresos para la plataforma iOS y el $80 \%$ en el caso de Android, son países de Asia Oriental y países europeos de habla no inglesa (Chan, 2014).

De los distintos dispositivos móviles, el teléfono inteligente ha sido el más empleado en el 2015 en España, según los datos ofrecidos por la Fundación Telefónica (2015). Esta supremacía viene de la mano de un incremento en la descarga de aplicaciones móviles. Según esta misma fuente, el número de unidades vendidas en el tercer trimestre de 2015 fue de 355, 2 millones, lo que superaba en un 6,8 $\%$ las unidades vendidas durante el mismo periodo en el 2014. En lo referente a las aplicaciones, y según la misma fuente, el dispositivo elegido a nivel mundial como fuente de descarga fue el teléfono móvil, seguido de las tabletas. Un 90 \% del tiempo de conexión está destinado al uso de aplicaciones móviles, y cada mes se lanzan al mercado unas 40.000 nuevas aplicaciones. En el caso de España, y en referencia al 2015, se observa "un total de 27,7 millones de usuarios de aplicaciones que se descargan 3,8 millones de aplicaciones diariamente. La media de aplicaciones por dispositivos es de 30 en los smartphones y de 24 en el caso de las tablets [...]". (Fundación Telefónica, 2015, p. 42). 
Llegado este punto, cabe plantearse los retos que la enseñanza de la localización de aplicaciones móviles supone. Distintos estudios han tratado de indagar en las principales diferencias entre traducción y localización y resaltar el lugar que ocupa la localización dentro de los estudios de traducción. Como bien señala Jiménez Crespo (2013), el proceso ha sido lento, pero hoy en día puede considerarse como una disciplina dentro de la Traducción. Ciertos trabajos se centran en definir el concepto de la localización (Pym, 2004), así como describir las destrezas necesarias que los traductores necesitan para trabajar en el campo de la localización (DiFranco, 2013). Otros trabajos se alejan del punto de vista más académico para acercarse al contenido más técnico de la localización (Roturier, 2015). A nuestro parecer, la principal diferencia entre localización y traducción se encuentra en el proceso de trasvase y en el producto final, junto con la diversidad de contenidos lingüístico-textual y no lingüístico, que juega un papel clave en el proceso de localización. De un lado, el proceso de trasvase en la localización está directamente imbricado con los aspectos técnicos, además de caracterizarse por un flujo de trabajo en constante cambio, que lo dota de dinamismo entre los distintos actores implicados (traductores, gestores de proyectos, ingenieros, etc.) y que se conoce como agile localization. Así, según el tipo de localización, se traducen y se adapta información diversa (lingüístico-textual, elementos multimedia y aspectos culturales) y formatos (.html, .xml, .svg, .po, etc.). Igualmente se puede llegar a trabajar con distintos lenguajes de programación, lo que va a requerir del traductor un conocimiento básico de los mismos. Tal es el caso, por ejemplo, de la localización de un sitio web, donde el traductor debe estar familiarizado con lenguajes de programación frecuentes en el diseño de sitios web dinámicos, como puede ser .php. Hay herramientas informáticas y programas especializados de localización que facilitan la tarea de trasvase, puesto que separan el texto traducible del código (Passolo, para el caso concreto de localización de programas informáticos), aunque también puede accederse de forma directa al código del producto digital. Sin embargo, tal y como señala Fernández Costales (2009), el conocimiento experto y las destrezas técnicas se hacen necesarias en estos casos. De otro, en lo referido al producto de la localización o el material localizado estamos frente a un producto digital que debe ser adaptado desde el punto lingüístico y cultural para satisfacer las necesidades de un mercado específico, un producto que, en la mayoría de los casos, es de naturaleza dinámica y se actualiza de forma constante.

A los retos que plantean el proceso y el producto en la localización en general, y que se encuentran de forma directa en la localización de aplicaciones móviles, hay que sumar retos de carácter pedagógico, como pueden ser las limitaciones técnicas (necesidad de programas informáticos específicos, aulas de informática equipadas), materiales de referencia, investigaciones previas, y las propias impuestas por los planes de estudios, donde el número de horas destinadas a asignaturas específicas de localización puede ser reducido, lo que dificulta la incorporación de otro tipo de localización a las ya conocidas. En otro sentido, no se puede obviar la necesidad de comunicación entre el mundo empresarial y el académico con el fin de satisfacer las necesidades del mercado y que nuestros alumnos desarrollen unas competencias que les permitan integrarse de forma satisfactoria en el mundo laboral de la localización. Dicha comunicación puede materializarse en programas formativos dentro del ámbito académico, como cursos impartidos por profesionales, además de un programa de prácticas en empresas de localización.

Como ocurre con los distintos tipos de localización, creemos que la localización de aplicaciones móviles, debido a que se trata de un fenómeno reciente, supone un reto adicional. Sánchez Ramos 
y Morado Vázquez (2015) señalan algunas de las principales dificultades que existen, como son las limitaciones de tamaño, ya que las aplicaciones móviles cuentan con un hardware y recursos de procesamiento mucho más reducidos que los programas informáticos tradicionales; el tiempo de instalación, que suele llevar minutos; la funcionalidad, es decir, la finalidad de uso de las mismas, que suele ser reducida; y, por último, las actualizaciones constantes.

De un lado, desde un punto de vista metodológico, la enseñanza de la localización debe abordarse de tal forma que el alumno adquiera un conocimiento procedimental y conceptual para así poder adquirir las destrezas y competencias técnicas necesarias que aseguren una inmersión adecuada en el mundo de la localización. A nuestro parecer, creemos que el enfoque adoptado por Folaron (2006, p. 212) aporta una aproximación metodológica clave para la enseñanza de la localización. Se trata de una aproximación centrada en el proceso y en consonancia con las expectativas de la industria de la localización, que se centra en tres aspectos clave: actuación, identificación de un problema y solución del mismo. Por otro lado, desde un punto de vista pedagógico, se hace necesaria la enseñanza de la localización de aplicaciones móviles desde una perspectiva que englobe desafíos de tipo lingüístico, técnico y cultural. Detallamos a continuación los más destacables.

\section{Elementos lingüísticos:}

- A diferencia de la localización de programas informáticos, donde el traductor se enfrenta a distintos elementos localizables en forma de archivos de código fuente o archivos binarios, en la localización de aplicaciones móviles suele trabajarse con archivos XML (ver Figura 1). El componente lingüístico-textual dependerá de la plataforma para la que se desarrolle la aplicación. Por ejemplo, Android hace uso de archivos XML, mientras que iOS lo hace con las llamadas strings. Tomando como ejemplo el caso de Android, este tipo de archivos suelen encontrarse en la carpeta res (recursos). Dentro de dicha carpeta se incluye la carpeta values,

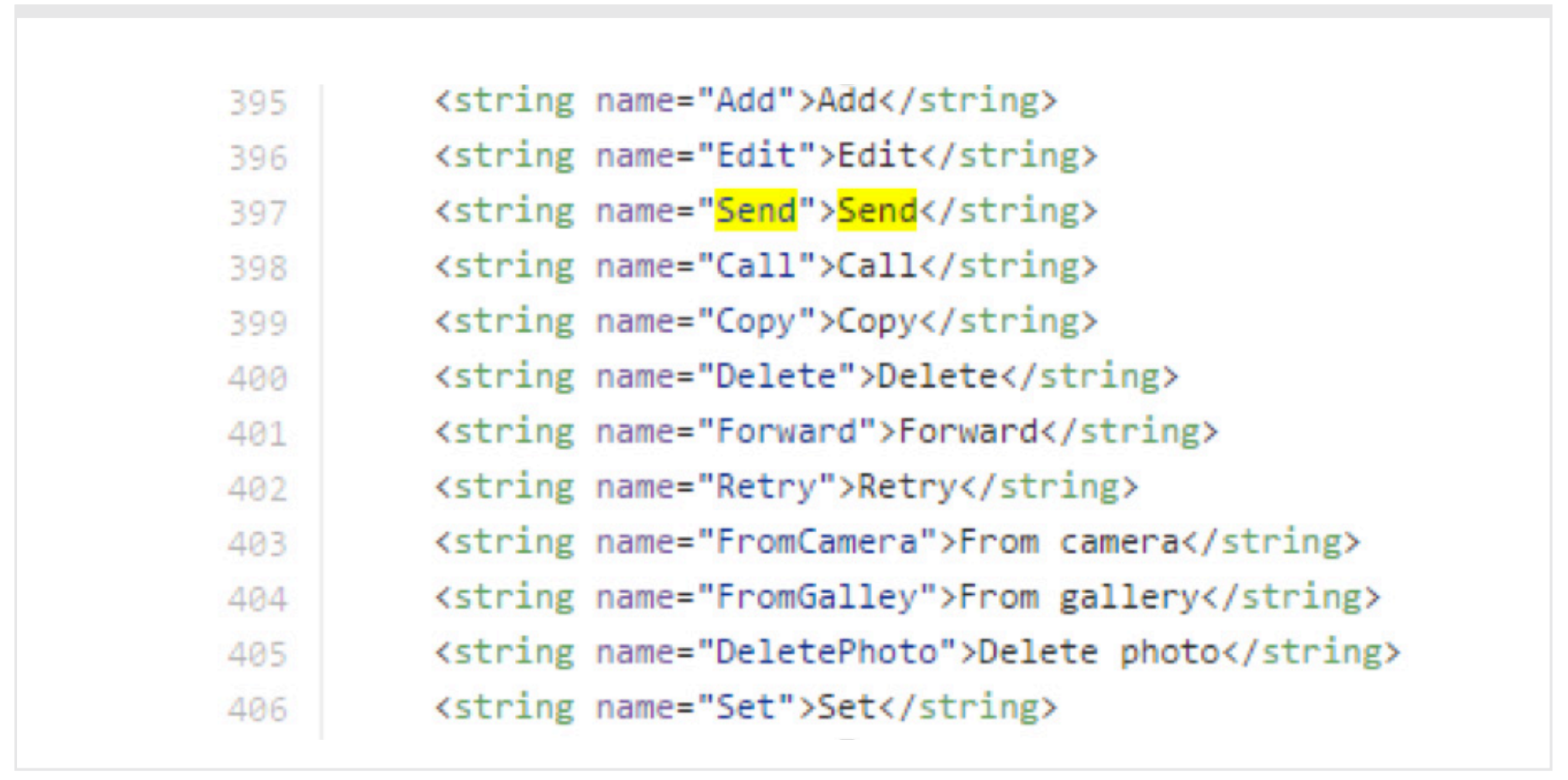

Figura 1. Ejemplo de código fuente extraído de la aplicación Telegram 
y dentro de la misma el archivo strings.xml, donde destaca el atributo y el texto de la cadena, y que será el archivo con el que normalmente trabaje el traductor. Normalmente este tipo de archivos pueden traducirse con una herramienta de traducción asistida (TAO).

- Registro: a la hora de localizar una aplicación móvil, hay que tener en cuenta los usuarios de la misma y parámetros como la edad, por ejemplo, harán que el traductor se decante por un tipo de registro. Será de los usuarios de quien dependa la traducción interlingüística y evitará las incoherencias de registro.

- Terminología: de nuevo será relevante tener en cuenta la plataforma para la que se ha desarrollado la aplicación (Android, iOS, Windows Phone). Hoy en día los distintos desarrolladores ofrecen guías de ayuda o incluso existen portales lingüísticos, como el de Microsoft, que facilitan las tareas de los traductores. La consistencia terminológica según el tipo de plataforma, por tanto, será de máxima importancia (Palomares, 2012).

- Contextualización: como se ha comentado anteriormente, se trabaja con cadenas de texto (strings), lo que en la mayoría de ocasiones dificultará la comprensión del contenido textual. La falta de contexto, puesto que el traductor suele recibir dichas cadenas, en algunos casos, en hojas de cálculo, es un añadido a la dificultad de la localización de aplicaciones móviles.

- Elementos multimedia: el empleo de elementos no verbales, como las imágenes, por ejemplo, favorecen la inserción de elementos intertextuales. No hay que olvidar que las aplicaciones móviles son, en su mayoría, productos comerciales y destinados al entretenimiento, con lo que el efecto visual es clave. Se trata, por tanto, de elementos localizables, donde además de la creatividad traductora, el localizador deberá considerar su adaptación lingüística y cultural. La mayoría de estos elementos multimedia suele incluir material lingüístico que el traductor deberá localizar.

\section{Elementos técnicos:}

- Limitaciones de espacio: aunque existe una amplia gama de tamaños de dispositivos móviles, es cierto que las dimensiones de la pantalla suelen ser reducidas, por lo que el elemento textual se ve igualmente reducido. Si bien este aspecto técnico responde al plano de la internacionalización como fase previa a la localización, es algo de lo que el localizador debe ser consciente y ceñirse, si no se especifica de otro modo, a no emplear traducciones largas.

- Herramientas de localización: al igual que ocurre en la localización de programas informáticos, el localizador puede trabajar con herramientas TAO', puesto que la gran mayoría reconoce la extensión .xml, y extraerá el texto traducible. No obstante, en algunos casos puede necesitar la creación de filtros. Otra opción es trabajar directamente con un editor de texto avanzado y el código, aunque no es lo más recomendable puesto que requiere un conocimiento del lenguaje de programación para no eliminar los códigos funcionales. Hay que subrayar que existe una 
escasez de programas específicos para la localización de aplicaciones móviles, al contrario de lo que ocurre con la localización de programas informáticos, que cuenta con herramientas específicas como SDL Passolo o Alchemy Catalyst.

\section{Elementos culturales:}

- Adaptación de formatos a cada mercado: igual que ocurre con la localización de programas informáticos, los calendarios, la moneda o las cifras deben adaptarse a la cultura meta.

- El elemento visual vuelve a destacar si hablamos de adaptación cultural. Podemos tomar como ejemplo la aplicación de mensajería KakaoTalk, donde el cambio de color en la interfaz de usuario (de amarillo a naranja), responde a cuestiones culturales y de adaptación a un mercado específico.

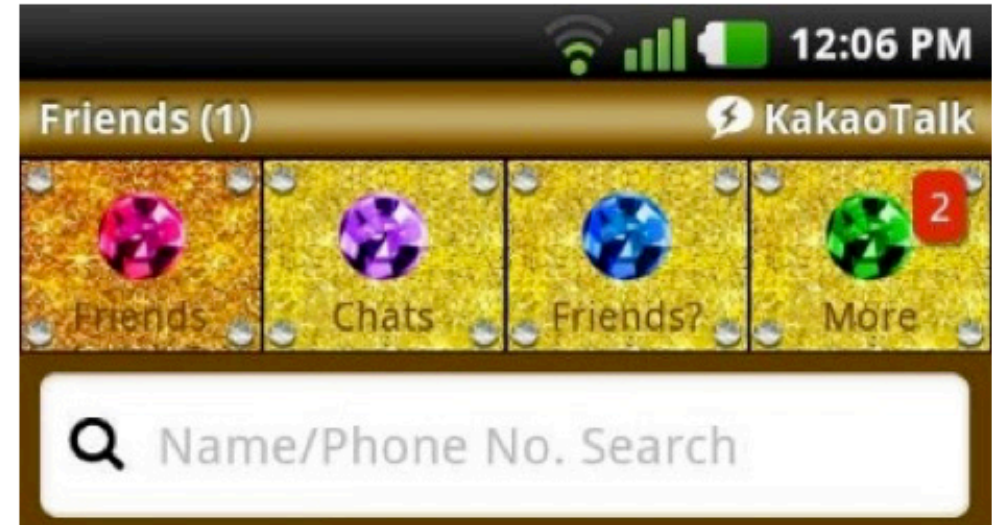

My Profile

\section{KakaoTalkThem...}

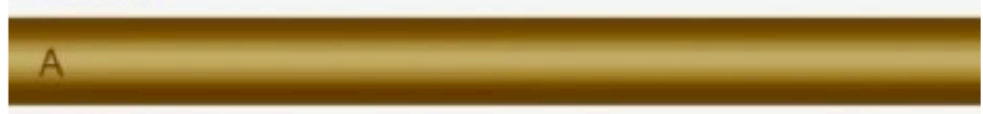

\section{AppsGraphics}

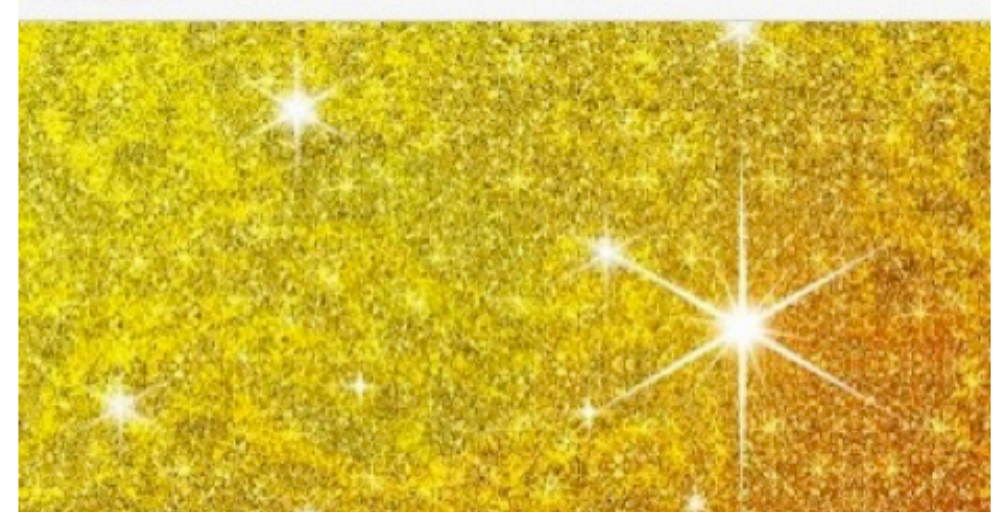

Figura 2. Interfaz KakaoTalk (Corea)

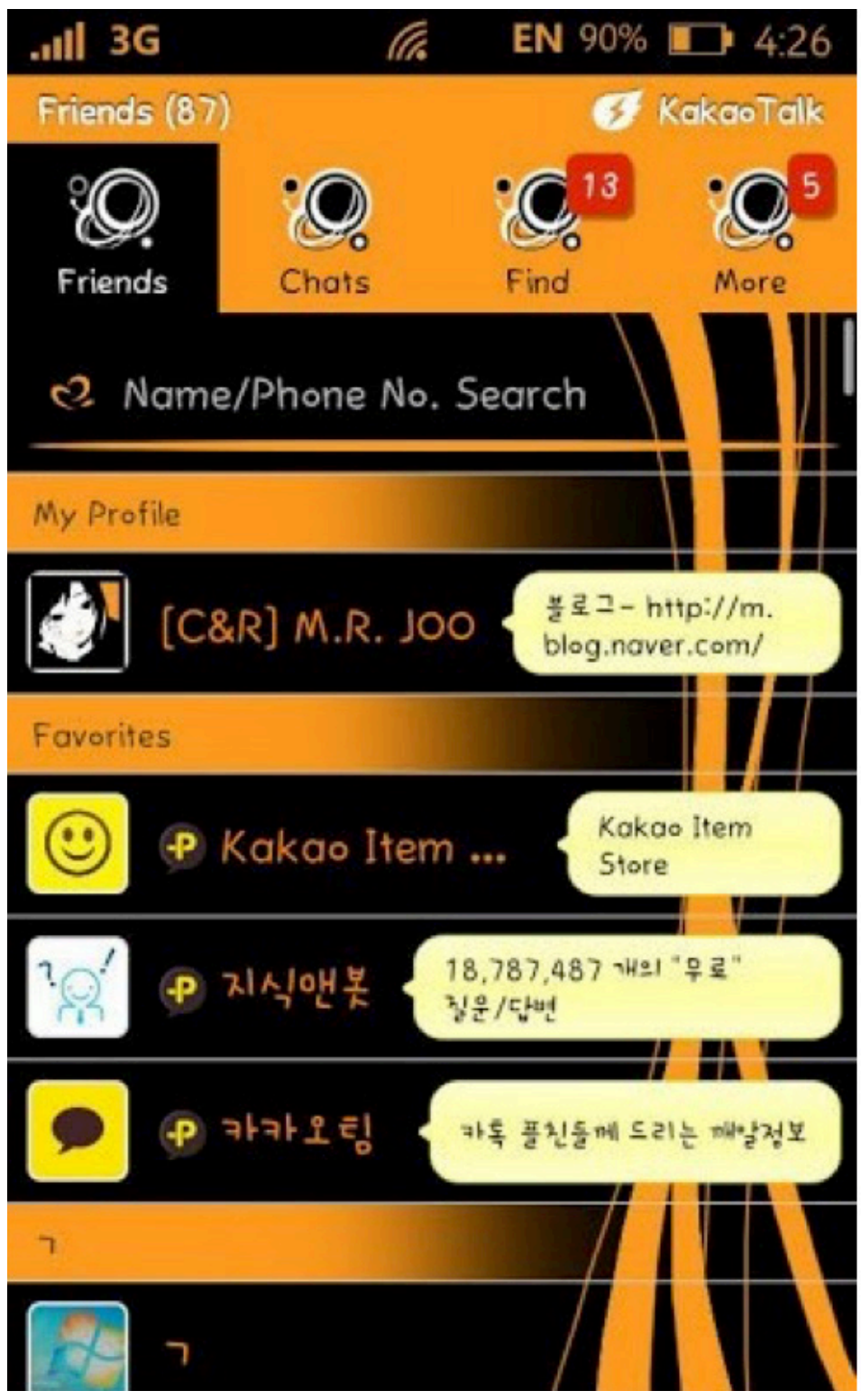

Figura 3. Interfaz KakaoTalk Indonesia 


\section{MÉTODO}

\section{Diseño}

El estudio se fundamentó siguiendo las bases de la enseñanza socioconstructivista (Kiraly, 2000), que aboga por prácticas traductológicas reales en forma de proyectos, como puede ser el caso de las tareas realizadas bajo los enfoques situacionales y el aprendizaje basado en proyectos (Moursund, 1999; Thomas, Mergendoller \& Michaelson, 1999), en los que el profesor se vuelve 'orquestador' y facilitador del aprendizaje. Este tipo de enseñanza conforma como una de las teorías más influyentes en la didáctica de la traducción. La teoría socioconstructivista promueve "teaching methods that foster responsibility, independence, and the ability to see alternatives" (Kiraly 2000, p. 33). Bajo esta teoría, la didáctica de la traducción adquiere su condición más dinámica y se dirige hacia la construcción de conocimiento. Parece lógico, pues, pensar que el enfoque socioconstructivista está íntimamente ligado a la filosofía de la era digital y la Web2.0, en donde los usuarios, además de subir contenidos y difundir la información a través de Internet, interactúan y 'colaboran' entre ellos (Desjardins, 2011, p. 179). Si trasladamos el enfoque socioconstructivista al aula de traducción, hay ciertos aspectos que deberán tenerse en cuenta. Será necesario un entorno físico apropiado, dotado de un marco de interacción, en el que el alumno, en su papel de traductor, trabaje e interactúe con otros participantes y maneje recursos tecnológicos y lingüísticos. Un buen entorno posibilitará la construcción del aprendizaje a través de la interacción y la responsabilidad entre los miembros del equipo (Robinson, López Rodríguez \& Tercedor Sánchez, 2008, p. 100). Las actividades diseñadas bajo este enfoque podrán basarse en tareas o bien en proyectos. Por otro lado, el aprendizaje basado en proyectos parece ofrecer un entorno oportuno en el que desarrollar una propuesta que simule las condiciones de trabajo en entornos profesionales, en nuestro caso, de trabajo colaborativo. Esta metodología ha sido utilizada en los contextos de la didáctica de la traducción, como es el caso de García González y Veiga Díaz (2015) o Galán Mañas (2011).

\section{Contexto}

El estudio realizado se enmarcó dentro del módulo dedicado a la localización de aplicaciones móviles, dentro de la asignatura de Localización, que se imparte en el Grado en Lenguas Modernas y Traducción de la Universidad de Alcalá (Madrid, España). Dicho módulo ocupa el último lugar en el sílabo, con lo que los alumnos cuentan con un bagaje teórico y técnico relacionado con la localización de programas informáticos y web y conceptos clave como lenguajes de etiquetado. La actividad comprende un total de tres horas presenciales. La puesta en práctica de la misma se realizó en una sesión de dos horas y la hora restante se dedicó a la exposición oral de los resultados por parte de los alumnos en una sesión conjunta. La actividad ofrecía al alumnado tres entornos (localización con un editor de texto avanzado, localización con una herramienta de traducción asistida y localización con programas concretos que permiten trabajar con archivos .apk) en los que podría llevarse la localización de una aplicación móvil con el principal objetivo de poder contrastar las ventajas y desventajas de cada uno de ellos. La tarea consistió en localizar una aplicación móvil de mensajería (Telegram) del inglés al español para Android, cuyo código fuente está disponible en Internet. La clase quedó dividida en seis grupos (dos grupos para cada uno de los tres entornos mencionados anteriormentel formados por cuatro alumnos cada uno, encargados de la traducción y revisión del producto final. Al finalizar la localización, cada grupo realizó una exposición oral del proceso de traducción, en donde se pidió una reflexión sobre las principales dificultades encontradas, las soluciones ofrecidas y una valoración final. Tras la exposición de cada grupo hubo un pequeño debate entre todos los grupos, guiado por el profesor, que sirvió de cierre para 
la actividad y de reflexión sobre las distintas herramientas. Los alumnos también elaboraron un dossier que recogía el proceso de localización con cada una de las herramientas, así como las dificultades encontradas y las soluciones propuestas. Para la puesta en marcha de la actividad e intercambio de archivos se utilizó la plataforma virtual Blackboard. Cada grupo subió su dossier a dicha plataforma para que así cada grupo tuviera acceso a los distintos procesos de localización.

\section{Participantes}

Los alumnos que participaron en el experimento fueron 24, todos matriculados en la asignatura optativa de Localización, y que habían cursado y aprobado la asignatura obligatoria de Herramientas informáticas aplicadas a la traducción.

\section{Instrumentos de recolección de datos}

Los datos obtenidos fueron de índole cualitativa. Para la recolección de datos se optó por dos herramientas. De un lado, la sesión en la que los grupos exponían sus resultados (exposición oral) junto con el dossier. El principal objetivo era el de otorgar al alumnado un papel principal, a la vez que diseñar la clase como un entorno de aprendizaje basado en el intercambio de ideas entre los sujetos. Tanto los grupos de trabajo iniciales como la exposición final fueron muy útiles a la hora de intercambiar ideas, problemas con las herramientas utilizadas, soluciones entre los grupos que compartían la misma herramienta, etc. El dossier jugó también un papel importante ya que sirvió para plasmar el proceso de localización y que el resto de los grupos tuviera acceso al mismo. La segunda herramienta fue un cuestionario con cinco preguntas (ver Apéndice A), donde los alumnos tuvieron la oportunidad de valorar la actividad en sí y el módulo específico sobre localización de aplicaciones móviles dentro de la asignatura de Localización. El profesor también formó parte en el proceso de recolección de datos: guiando la exposición oral y proporcionando pautas para el desarrollo de la misma.

\section{Procedimiento}

Como ha quedado descrito anteriormente, la distribución del alumnado se hizo en seis grupos formados por cuatro alumnos. Grupos 1 y 2 se encargarían de localizar la aplicación móvil con un editor de texto avanzado; grupos 3 y 4 lo harían con una herramienta TAO'; y los grupos 5 y 6 lo harían con herramientas más enfocadas a la localización de aplicaciones móviles.

En el caso de los grupos 1 y 2, el profesor proporcionó a los alumnos el material necesario para realizar la actividad: el proyecto Telegram, que incluye el código fuente, una carpeta de recursos y las llamadas values, que recogen las cadenas de texto en .xml que deberán traducir. Como herramientas se sugiere utilizar algunos de los más conocidos editores de texto avanzado y que se encuentran instalados en el aula de informática, como Notepad++ o PsPad.

Para los grupos 3 y 4 , al igual que ocurre con los grupos anteriores, el alumno cuenta con el código fuente y las cadenas de texto en $\mathrm{xml}$, un formato que puede utilizarse en herramientas TAO como OmegaT o SDL Trados en cualquiera de sus versiones. 
Por último, para los grupos 5 y 6, el profesor proporciona el archivo .apk de la aplicación. El alumno cuenta también con material sobre la localización de aplicaciones móviles con programas como APK tools o MultiTools. Tras la localización de la aplicación se procede a la exposición oral y reflexión grupal de las tareas.

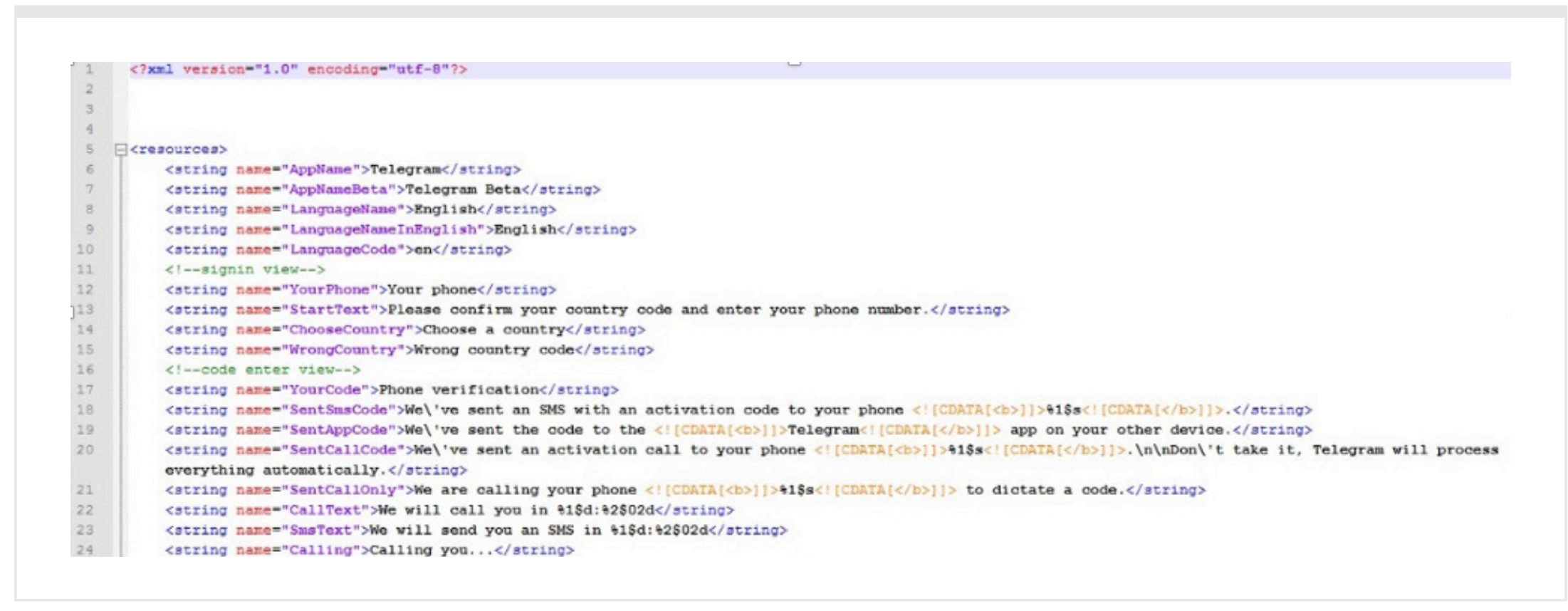

Figura 4. Cadenas de texto en un editor de texto avanzado

\section{RESULTADOS}

Los resultados obtenidos tanto de la exposición oral como del cuestionario final de evaluación sirvieron para, de un lado, establecer un debate sobre las distintas formas de enfrentarse a la localización de una aplicación móvil, así como de las ventajas y desventajas que cada procedimiento puede acarrear. Por otro lado, el cuestionario fue de gran utilidad para evaluar la propuesta formativa, el cual arrojó luz sobre la actitud de los discentes hacia este tipo de localización y la propuesta docente.

En lo referido a la exposición oral, los grupos 1 y 2 destacaron la facilidad a la hora de traducir las cadenas de texto con un editor de texto avanzado. No obstante, a pesar de la mínima dificultad a la hora de traducir, fueron conscientes de los problemas que podían presentarse. Entre ellos destacaban la falta de contexto, la imposibilidad de utilizar una memoria de traducción o la necesidad de estar familiarizado con el lenguaje .xml para no dañar el código. Si bien en un principio parecía la opción más sencilla a la hora de traducir una aplicación móvil, tras la exposición y la reflexión final con el resto de los grupos quedó prácticamente descartada. Reproducimos algunos de los comentarios efectuados por los alumnos en el dossier sobre las ventajas y desventajas a la hora de utilizar un editor de texto avanzado:

- Es complicado pasar de un segmento a otro, ya que hay que hacerlo con el teclado y se pierde tiempo. Hay que seleccionar de forma manual las partes que se van a traducción. Además, [el editor de texto avanzado] Notepadd ++ no separa el texto traducible del que no lo es.

- Es muy fácil pasar de largo los segmentos que hay que traducir y perderse en el código.

- Como punto a favor del editor tengo que decir que es un programa fácil de usar. Eso sí, hay que tener unos conocimientos del lenguaje XML. 
Los grupos 3 y 4 emplearon SDL Trados 2014 para realizar la traducción de las cadenas de texto. La discusión grupal fue altamente satisfactoria, puesto que todos los alumnos coincidieron en las facilidades de una herramienta de TAO, puesto que el código está protegido y se pueden emplear memorias de traducción. Sin embargo, requiere también de conocimientos técnicos puesto que, en ocasiones, se hace necesaria la creación de filtros y conocimiento de lenguaje xml y expresiones regulares para la creación de dichos filtros. Tal es el caso de la aplicación propuesta, que, como puede verse en la figura 5, necesita la creación de filtros para 'limpiar' el texto meta.

\begin{tabular}{|c|c|c|c|}
\hline & \multirow[b]{2}{*}{$\mathrm{XML}:$ Any $\vee 12.0 .0$} & & \multirow{3}{*}{ XML: Any v 1.2.0.0 } \\
\hline & & & \\
\hline 1 & Telegram & $\bar{z}$ & \\
\hline 2 & Telegram Beta & $\neg$ & \\
\hline 3 & English & ב & \\
\hline 4 & English & ב & \\
\hline 5 & en & - & \\
\hline 6 & Your phone & ב & \\
\hline 7 & Please confirm your country code and enter your phone number. & Z & \\
\hline 8 & Choose a country & ב & \\
\hline 9 & Wrong country code & ב & \\
\hline 10 & Phone verification & ב & \\
\hline 11 & Welve sent an SMS with an activation code to your phone & $\bar{Z}$ & \\
\hline 12 & $<\mathrm{b}>$ & ב & \\
\hline 13 & $\% 1 \$ s$ & 7 & \\
\hline 14 & $\langle/ b\rangle$ & ב & \\
\hline 15 & . & ב & \\
\hline 16 & Welve sent the code to the & ב & \\
\hline 17 & $\langle h\rangle$ & 7 & \\
\hline
\end{tabular}

Figura 5. Cadenas de texto en una herramienta TAO

\section{Algunos de los comentarios de los alumnos fueron:}

- La traducción sencilla y rápida, ya que es una herramienta que sabemos utilizar y nos permite reutilizar otras traducciones en forma de memorias de traducción.

- Es necesario crear un proyecto de traducción, pero es fácil y no lleva mucho tiempo.

- Al finalizar el proceso de traducción, hay que reemplazar en la carpeta el archivo original con la traducción o no funcionará aplicación en el idioma traducido.

- La herramienta TAO elimina la mayor parte de las etiquetas, con lo que nos evitamos dañar el código.

Por último, los grupos 5 y 6 concluyeron que el uso de programas específicos para la localización de aplicaciones móviles parecía una solución adecuada, aunque suelen aparecer problemas de naturaleza técnica. En este tercer caso, además de necesitar conocer el lenguaje xml y el uso de herramientas TAO (la traducción de las cadenas de textos se puede hacer con dichas herramientas), se precisan otros conocimientos técnicos a la hora de la instalación de dichos programas y el manejo de otro tipo de formatos como los archivos de aplicación .apk. 


\section{En este caso, destacamos los siguientes comentarios:}

- Se han encontrado problemas técnicos que han hecho que el proceso de traducción sea lento.

- Además de conocer la lengua, se precisan conocimientos técnicos y de manejo de las herramientas ...y paciencia.

- Creo que las principales desventajas son: el tiempo que tarda en instalarse, tanto el entorno como los componentes necesarios para que funcione bien y la cantidad de dificultades técnicas a las que ha habido que hacer frente lerrores a la hora de abrir la aplicación en el entorno, errores a la hora de crear un emulador con BlueStacks, errores a la hora de intentar ejecutar la aplicación...).

- Permite comprobar el funcionamiento de la aplicación a través de un emulador.

Junto con la recogida de datos procedentes de la exposición oral y el dossier, y para apoyar los datos obtenidos, se procedió a la distribución de un cuestionario final para evaluar la propuesta docente. Así, los alumnos tuvieron la oportunidad de valorar dicha experiencia a través de un breve cuestionario formado por un total de cinco preguntas (cuatro preguntas cerradas y una abierta). La pregunta 1 (¿Consideras que este módulo ha sido necesario para completar tu visión de localización?) era una pregunta cerrada, donde los sujetos tenían que seleccionar Sí o No como respuesta. Todos los sujetos (24) seleccionaron la opción Sí, lo que demuestra la buena acogida que tuvo el módulo sobre localización de aplicaciones móviles. Hay que señalar que este módulo ocupó una posición final en el sílabo, con lo que los alumnos contaban ya con un manejo considerable de herramientas de localización y conocimientos técnicos necesarios para enfrentarse a dichas tareas.

La Pregunta 2 recogía información sobre la utilidad de la actividad planteada, donde destaca que para la mayoría del alumnado (22 alumnos), la experiencia docente ha sido muy útil.

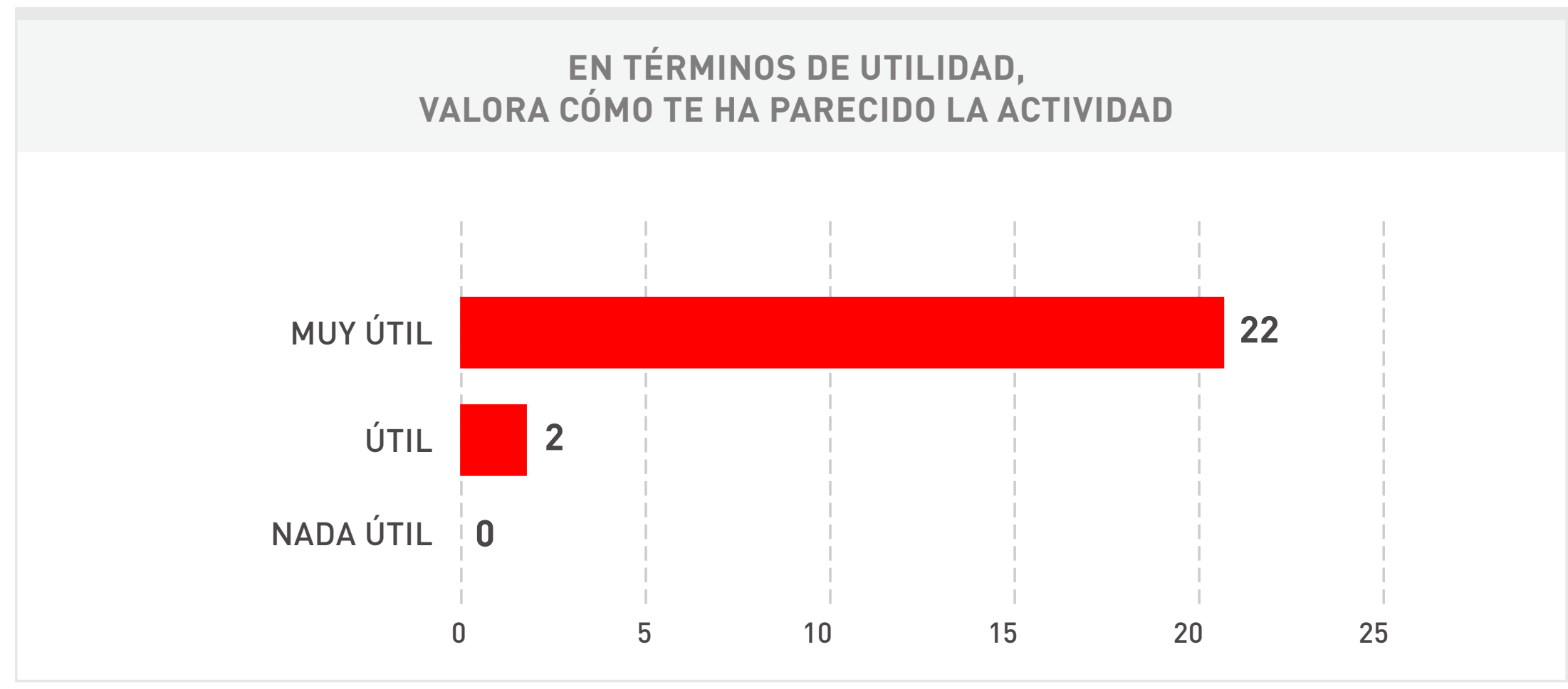

Figura 6. Resultados Pregunta 2 
En la tercera pregunta ¿¿Cómo consideras la estructura de la actividad? los alumnos valoraban la idoneidad de la organización de la actividad (organización grupal, exposición y dossier). Con esta metodología se buscaba una mayor interacción entre el alumnado y una disposición de la clase, en forma de panel de exposiciones y debate, que fomentara el intercambio de ideas y ayudara a solventar los problemas encontrados. Los tres contextos que se propusieron en la actividad no eran ajenos a ningún grupo, con lo que no se privó a los alumnos de ninguno de ellos. A tenor de los resultados obtenidos (Muy adecuada, 83,3 \%; Adecuada 16,6 \% y Nada adecuada, $0 \%$ ), se deduce que el alumnado quedó satisfecho con este tipo de metodología.

Muy relacionada con la anterior, la pregunta número 4 volvía a confirmar la satisfacción del alumnado con la metodología realizada. Sin duda, a esta satisfacción contribuyó las instrucciones dadas a cada grupo sobre las distintas tareas para realizar tanto en la exposición como en la elaboración del dossier.

\section{¿CREES QUE LA EXPOSICIÓN Y EL DOSSIER DE TUS COMPAÑEROS HA SIDO SUFICIENTE PARA COMPRENDER EL PROCEDIMIENTO?}

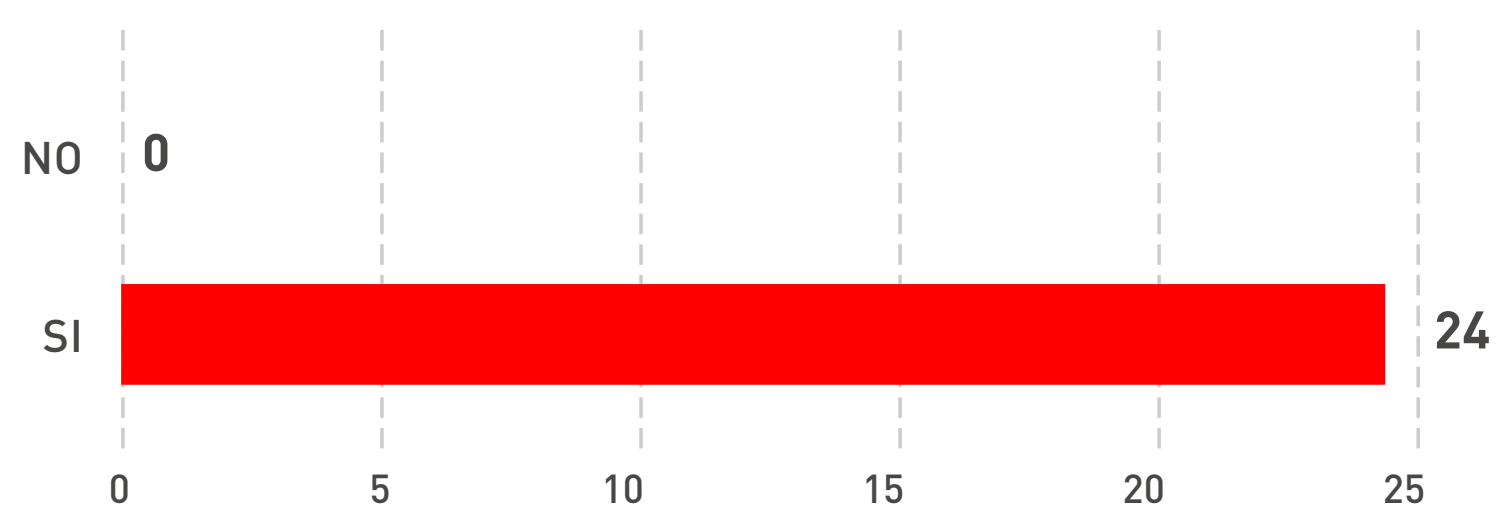

Figura 6. Resultados Pregunta 4

Por último, la pregunta número 5 era abierta y se pedía a los alumnos que indicaran cualquier comentario o sugerencia sobre la actividad. La mayoría de ellos coincidieron con los comentarios puestos ya de manifiesto en la exposición y que destacaban el proporcionar distintos entornos en las tareas de localización, además de resaltar la necesidad de dedicar más tiempo a la localización de aplicaciones móviles dentro de la asignatura de localización.

\section{DISCUSIÓN}

Tanto desde un punto de vista de los proveedores de los servicios lingüísticos, como desde la formación (Valero Garcés \& Toudic, 2015), la industria de la localización sigue su continuo ascenso. Nuevas modalidades de localización nos advierten de este avance, como la localización de aplicaciones móviles. Si bien se abren nuevas posibilidades para nuestros egresados, se generan desafíos en la enseñanza de la localización en el aula de traducción. 
Desde este trabajo nos hemos planteado conocer las dificultades que la enseñanza de la localización de aplicaciones móviles puede suponer en el aula de traducción. Aunque este tipo de localización comparte características con la localización de programas informáticos, su investigación en el aula de traducción, así como materiales de referencias o programas informáticos específicos resulta escasa (Sánchez Ramos \& Morado Vázquez, 2015). Es por ello que se proponen los distintos factores (lingüísticos, técnicos y culturales) que, a nuestro juicio, deben tenerse en cuenta en la didáctica de la localización de aplicaciones móviles.

A la vista del crecimiento del desarrollo de aplicaciones móviles y de la demanda de esta localización en el mundo profesional, y como parte de la asignatura de Localización, se creyó conveniente señalar' la propuesta didáctica que se realizó en el aula de traducción y que sirve como propuesta inicial para abordar la enseñanza de localización de aplicaciones móviles. Con el fin de contribuir a las distintas propuestas existentes que abogan por incluir la localización dentro del aula de traducción (Altanero, 2006; Baños \& Toto, 2013), nuestro trabajo ha descrito la puesta en práctica de una propuesta docente centrada en la enseñanza de la localización de aplicaciones móviles, a la vista de la mayor demanda que la industria de la localización de profesionales especializados y la llamada de estudiosos que piden una mayor atención a contenidos didácticos que desarrollen las competencias necesarias por parte de nuestros egresados (Dunne, 2006; Veiga Díaz, 2013). Los datos cualitativos obtenidos, procedentes de una exposición oral, dossier y cuestionario final de evaluación, indican una actitud positiva por parte del alumnado hacia la propuesta docente, así como la estructura y organización de la misma, y la necesidad de este tipo de actividades. Con todo, y a la luz de la experiencia docente, consideramos que, sin olvidar las limitaciones que puedan observarse de nuestra propuesta, nuestra experiencia docente puede servir de punto de partida para futuros docentes interesados en incluir nuevas prácticas relacionadas con la localización.

\section{REFERENCIAS}

Altanero, T. (2006). The localization job market in academe. En A. Pym, A. Perekresenko \& B. Starik (Eds.), Translation Technology and its Teaching (pp. 31-28). Tarragona: Servei de Publicacions.

Baños, R. \& Toto, A. (2013). Challenges and constraints in designing a localisation module for a multilingual cohort. En P. Sánchez Gijón, 0.

Torres-Hostench \& B. Mesa Lao (Eds.). Conducting Research in Translation Technologies (pp. 185-205). Berna: Peter Lang.

Chan, V. (2014). Compelling Statistics About the Importance of Localization. Recuperado de https://goo.gl/9C1Hj1

Desjardins, R. (2011). Facebook me! Initial insights in favour of using social networking as a tool for translator training. Linguistica Antverpien-

sia, 10, 175-192.

Drugan, J. (2013). Quality in Professional Translation. Assessment and Improvement. Londres: Bloomsbury.

DiFranco, C. (2003). Localization skills for translators: localizability requirements for clients. The Ata Chronicle, 32(1), 52-57.

Dunne, K. (2006). Perspectives on Localization. Ámsterdam: John Benjamins.

Esselink, B. (2000). A Practical Guide to Localization'. Ámsterdam: John Benjamins. 
Fernández Costales, A. (2009). Translation 2.0. The localization of institutional websites under the scope of functionalist approaches. En D. De Crom (Ed), Translation and the (Trans)formation of Identities. Selected Papers of the CETRA Research Seminar in Translation Studies 2008. Recuperado de https://goo.gl/xDsa8W

Folaron, D. (2006). A discipline coming of age in the digital age. En K. Dunne (Ed.), Perspectives on Localization (pp. 195-219). Ámsterdam: John Benjamins.

Fundación Telefónica. (2015). La Sociedad de la Información en España 2015. Barcelona: Ariel.

Galán Mañas, A. (2011). Translating authentic technical documents in specialized translation classes. JosTrans: The Journal of Specialized Translation, 16, 109-125

García González, M. \& Veiga Día, M. T. (2015). Guided inquiry and project-based learning in the field of specialised translation: a description of two learning experiences. Perspectives: Studies in translatology, 23(1), 107-123. doi: https://doi.org/10.1080/0907676X.2014.948018

Guevara Soriano, A. (2010). Dispositivos móviles. Revista Seguridad. Defensa Digital, 7. Recuperado de https://goo.gl/Ccu4r2

Jiménez Crespo, M. A. (2013). Translation and Web Localization. Londres y Nueva York: Routledge.

Kiraly, D. (2000). A Social Constructivist Approach to Translator Education: Empowerment from Theory to Practice. Manchester: St. Jerome.

Lachat Leal, C. (enero 2015). Localización de aplicaciones: estrategias de cooperación con los desarrolladores. Comunicación presentada en Asociación Ibérica de Estudios de Traducción e Interpretación (AIETI), Málaga, España. Recuperado de https://goo.gl/aGfMnx

Mata Pastor, M. (2005). Localización y traducción de contenido web. En D. Reineke (Ed). Traducción y Localización. Mercado, Gestión y Tecnologías (pp. 187-252). Las Palmas de Gran Canaria: Anroart Ediciones.

Moursund, D. (1999). Project-based Learning Using Information Technology. Eugene, OR: International society for technology in education.

Palomares, J. (2012), Mobile Apps L10N: The Basics. Recuperado de https://goo.gl/yqUrrM

Pym, A. (2004). The Moving Text : Localization, Translation and Distribution. Ámsterdam : Johns Benjamins.

Ray, R. (abril 2016). Preparing your team to localize more mobile moments. Multilingual. Recuperado de https://goo.gl/BU4uDF

Robinson, B., López Rodríguez, C. \& Tercedor Sánchez, M.I. (2008). Neither born nor made, but socially constructed: promoting interactive learning in an online environment. TTR: traduction, terminologie, redaction, 21(2), 95-129.

Roturier, J. (2015). Localizing Apps. Londres y Nueva York: Routledge.

Sánchez Ramos, M. M \& Morado Vázquez, L. (julio 2015). Locating' mobile localisation into the translator training curriculum. Ponencia presentada en V IATIS International Association of Translation and Interpreting Studies, Universidad Federal de Minas Gerais (UFMG), Belo Horizonte, Brasil.

Schäler, R. (2011). Localization. En M. Baker \& G. Saldanha. (Eds), Encyclopedia of Translation Studies (2nd ed.) (pp. 157-161). Londres y Nueva York: Routledge.

Schäler, R. (junio 2012). Mobile application development and Localisation. Taller presentado en LRC $10^{\text {th }}$ Annual Summer School, Mobile Application Development \& Localisation, Localisation Research Centre, University of Limerick, Irlanda.

Somers, H. (2003). Computers and Translation: A Translator's Guide. Ámsterdam: John Benjamins.

Thomas, J. W., Mergendoller, J. R., \& Michaelson, A. (1999). Project-based Learning: A Handbook for Middle and High School Teachers. Novato, CA: The Buck Institute for Education.

Valero-Garcés, C. \& Toudic, D. (2015). Technological innovation and translation. Training Translators in the EU for the $21^{\text {st }}$ century. Verbeia, 0 , 183-202. Recuperado de https://goo.gl/adA6fS

Veiga Díaz, M. T. (2013). La formación especializada en el nivel de posgrado en España: másteres y doctorados en traducción con componente tecnológico. Revista Tradumática, 11, 313-325.

(c) Los autores. Este artículo es publicado por la Revista Digital de Investigación en Docencia Universitaria del Área de Institutional Research and Effectiveness de la Dirección de Aseguramiento de la Calidad, Universidad Peruana de Ciencias Aplicadas. Este es un artículo de acceso abierto, distribuido bajo los términos de la LicenciaCreativeCommons Atribución-Compartirlgual 4.0 Internacional.l http://creativecommons.org/licenses/bysa/4.0/), que permite el uso no comercial, distribución y reproducción en cualquier medio, siempre que la obra original sea debidamente citada. 


\section{APÉNDICE A}

\section{CUESTIONARIO}

1. ¿Consideras que este módulo ha sido necesario para completar tu visión de la localización? $\int \mathrm{Si}$ No

2. En términos de utilidad, valora cómo te ha parecido la actividad

Muy útil

$\bigcirc$ Útil Nada útil

3. ¿Cómo consideras la estructura de organización de la actividad?

Muy adecuada

Adecuada

Nada adecuada

4. ¿Crees que la exposición y el dossier de tus compañeros ha sido suficiente para comprender el procedimiento de localización?

Sí

No

5. Añade cualquier comentario que creas conveniente.

Muchas gracias por responder a estas preguntas. 